\title{
On a Small Gain Theorem for Networks of iISS Systems
}

\author{
Hiroshi Ito, Sergey Dashkovskiy and Fabian Wirth
}

\begin{abstract}
This paper considers networks consisting of integral input-to-state stable (iISS) subsystems and addresses the problem of verifying iISS property of a given network. First, we focus on construction of continuously differentiable Lyapunov functions, and derive a condition ensuring the iISS of the network comprising $n$ subsystems. Although this approach referred to as the sum-type construction has not yet been reduced to an easily computable condition for general $n$, the $n=2$ case recovers the iISS small-gain condition for two subsystems developed recently. Next, in the case of $n$ subsystems, using Lipschitz continuous Lyapunov functions, this paper derives a small-gain condition. It is shown that this second approach referred to as the max-type construction fails to offer a Lyapunov function if there exist subsystems which are not input-to-state stable (ISS). The relation between the two formulations is discussed in the case of two ISS subsystems.
\end{abstract}

\section{INTRODUCTION}

When we establish stability of an interconnected system, the notion of input-to-state stability (ISS) is useful for dealing with the subsystems which do not admit a finite linear gain[19]. For verifying the ISS property of interconnection of two ISS subsystems, the ISS small-gain theorem is available in the literature[16], [22]. Since there are nonlinear systems which are not finite in the sense of ISS, the notion of integral input-to-state stability (iISS) has been developed to cover a class of such systems, [2]. For interconnection of two subsystems, the philosophy of the ISS small-gain theorem has been extended to the iISS case, [11], [14]. Needless to say, many practical systems such as logistic systems, biological systems, communication networks and power networks consist of more than two subsystems. Repeated application of the two systems argument to such large networks needs manipulations which cause unnecessary conservativeness. Recently, the ISS small-gain theorem has been extended to the case of general networks[7], [17].

The ISS small-gain theorem was originally given in terms of bounds for trajectories. Having Lyapunov functions is sometimes advantageous in analysis and design of nonlinear systems. A Lyapunov formulation of the ISS small-gain theorem was given in [15] for the first time, and extended to the general networks in [6], [8], [17]. The ISS Lyapunov

The work is supported in part by Grant-in-Aid for Scientific Research of JSPS under grant 19560446, by German Research Foundation (DFG) as part of the Collaborative Research Center 637, and by Volkswagen-Stiftung under grant I/82683-684.

H. Ito is with the Department of Systems Design and Informatics, Kyushu Institute of Technology, 680-4 Kawazu, Iizuka 820-8502, Japan hiroshi@ces.kyutech.ac.jp.

S. Dashkovskiy is with the Department of Mathematics and Computer Science, University of Bremen, Germany, dsnemath. uni-bremen. de.

F. Wirth is with the Institute of Mathematics, University of Würzburg, Germany, wirth@mathematik.uni-wuerzburg.de. functions constructed there are defined as the maximum among ISS Lyapunov functions of the subsystems, which directly yield Lipschitz continuous Lyapunov functions of the networks. In contrast, the iISS small-gain theorem developed in [11], [14] is proved by using the sum of iISS Lyapunov functions of the subsystems, which directly results in continuously differentiable Lyapunov functions. For such sum-type Lyapunov functions, no condition for establishing the stability of general networks is known. Although it can be mentioned that a sufficient condition and a corresponding sum-type Lyapunov function have been derived for interconnection consisting of $n$ ISS subsystems in [10], the result only deals with networks in a restricted structure defined as cascades of star products.

For the sake of obtaining smooth Lyapunov functions for the general networks consisting of ISS subsystems, an attempt has been made in [5] recently. Although smooth Lyapunov functions have not been obtained there for the ISS network, the study has made an important step which derives the max-type formula of Lyapunov functions from the ISS property of the subsystems in the dissipative form. Note that the max-type construction was originally derived from the ISS in the so-called implication form[15], [6], [8].

The purpose of this paper is to deal with subsystems described by dissipative inequalities covering the iISS property, and to elucidate capabilities, limitations and relations of two constructions. This paper shows that the max-type construction provides us with a small-gain condition for general $n$ subsystems. From the sum-type construction this paper also derives a sufficient condition for the stability of the network. Although the condition has not yet been computationally convenient for general $n$, it can be reduced to a small-gain condition in the case of two subsystems. Moreover, this paper demonstrates that the max-type construction can only deal with ISS subsystems. while the sum-type construction can handle iISS as well as ISS subsystems.

We use the following notation. The symbol $|\cdot|$ stands for the Euclidean norm. A continuous function $\omega: \mathbb{R}_{+}:=$ $[0, \infty) \rightarrow \mathbb{R}_{+}$is said to be positive definite and denoted by $\omega \in \mathcal{P}$ if it satisfies $\omega(0)=0$ and $\omega(s)>0$ holds for all $s>0$. A function is of class $\mathcal{K}$ if it belongs to $\mathcal{P}$ and is strictly increasing; of class $\mathcal{K}_{\infty}$ if it is of class $\mathcal{K}$ and is unbounded. The symbol Id denotes the identity maps. The symbols $\vee$ and $\wedge$ denote logical sum and logical product, respectively. Negation is $\neg$. For $f, g: \mathbb{R}_{+} \rightarrow \mathbb{R}_{+}$, we use the simple notation $\lim f(s)=\lim g(s)$ to describe $\{\lim f(s)=\infty \wedge \lim g(s)=\infty\} \vee\{\infty>\lim f(s)=$ $\lim g(s)\}$. Note that the $\infty$ case is included. In a similar manner, $\lim f(s) \geq \lim g(s)$ denotes $\{\lim f(s)=\infty \vee \infty>$ $\lim f(s) \geq \lim g(s)\}$. For vectors $a, b \in \mathbb{R}^{n}$ the relation $a \geq b$ is defined by $a_{i} \geq b_{i}$ for all $i=1, \ldots, n$. The relations 
$>, \leq,<$ for vectors are defined in the same manner. The negation of $a \geq b$ is denoted by $a \geq b$ and this means that there exists an $i \in\{1, \ldots, n\}$ such that $a_{i}<b_{i}$. For a function of time $t$, a dot over its symbol stands for $d / d t$.

\section{Problem Statement}

Consider a network $\Sigma$ whose state vector $x(t)=$ $\left[x_{1}(t)^{T}, x_{2}(t)^{T}, \ldots, x_{n}(t)^{T}\right]^{T} \in \mathbb{R}^{N}$ is governed by $\dot{x}=$ $f(x, r)$ and admits the existence of a positive definite and radially unbounded $\mathbb{R}_{+}$-valued function $V_{i}\left(x_{i}\right)$ satisfying

$$
\dot{V}_{i}\left(x_{i}\right) \leq-\alpha_{i i}\left(V_{i}\left(x_{i}\right)\right)+\sum_{j \neq i} \gamma_{i j}\left(V_{j}\left(x_{j}\right)\right)+\gamma_{r, i}(|r|)
$$

along the trajectories $x_{i}(t) \in \mathbb{R}^{N_{i}}$ for each $i=1,2, \ldots, n$. The vector $r(t) \in \mathbb{R}^{M}$ denotes an exogenous signal. The property (1) is usually called a dissipation inequality of $\Sigma_{i}$. It is assumed that $\alpha_{i i} \in \mathcal{K}, \gamma_{i j} \in \mathcal{K} \cup\{0\}$ and $\gamma_{r, i} \in \mathcal{K} \cup\{0\}$ hold. This assumption means that each subsystem $\Sigma_{i}$ defined with the state $x_{i}$ and the inputs $x_{j}$, $j \neq i, r$ is integral input-to-state stable (iISS), and that $V_{i}$ is an iISS Lyapunov function for the individual subsystem $\Sigma_{i}$ for each $i=1,2, \ldots, n$. We borrow the notions of ISS and iISS properties from the references[19], [21], [2]. Under a stronger assumption $\alpha_{i} \in \mathcal{K}_{\infty}$, the system $\Sigma_{i}$ is input-to-state stable (ISS), and the function $V_{i}$ is an ISS Lyapunov function. By definition, an ISS system is always iISS. The converse does not hold. The original definition of iISS and ISS is given in terms of trajectories, which is equivalent to the existence of $\mathcal{C}^{1}$ iISS and ISS Lyapunov functions, respectively[2], [21].

Remark 1: The function $V_{i}$ satisfying (1) is said to be an iISS Lyapunov function even when $\alpha_{i i} \in \mathcal{P}$ [2]. Nevertheless, to allow for feedback loops in the network $\Sigma$, this paper assumes $\alpha_{i i} \in \mathcal{K}$ which is a strict subset of $\mathcal{P}$. It is stressed that a feedback loop of iISS systems defined with dissipation inequalities (1) is iISS only if $\alpha_{i i} \in \mathcal{K}$ [12].

The objective of this paper is to derive conditions under which the network $\Sigma$ in total is iISS with respect to input $r$ and state $x$. We want to cover ISS as a special case. To this end, we define operators $A, \Gamma: s \in \mathbb{R}_{+}^{n} \mapsto z \in \mathbb{R}_{+}^{n}$ by

$$
\begin{aligned}
& z=A(s)=\left[\alpha_{11}\left(s_{1}\right), \alpha_{22}\left(s_{2}\right), \ldots, \alpha_{n n}\left(s_{n}\right)\right]^{T} \\
& z=\Gamma(s)=\left[\sum_{j \neq 1} \gamma_{1 j}\left(s_{j}\right), \sum_{j \neq 2} \gamma_{2 j}\left(s_{j}\right), \ldots, \sum_{j \neq n} \gamma_{n, j}\left(s_{j}\right)\right]^{T}
\end{aligned}
$$

The operator $\Gamma_{r}: \tau \in \mathbb{R}_{+} \mapsto z \in \mathbb{R}_{+}^{n}$ is defined by

$$
z=\Gamma_{r}(\tau)=\left[\gamma_{r, 1}(\tau), \gamma_{r, 2}(\tau), \ldots, \gamma_{r, n}(\tau)\right]^{T}
$$

The following vectors are also defined:

$$
\begin{aligned}
V(x) & =\left[\begin{array}{lll}
V_{1}\left(x_{1}\right), & V_{2}\left(x_{2}\right), \ldots, & V_{n}\left(x_{n}\right)
\end{array}\right]^{T} \\
\dot{V}(x) & =\left[\begin{array}{lll}
\dot{V}_{1}\left(x_{1}\right), & \dot{V}_{2}\left(x_{2}\right), \ldots, & \dot{V}_{n}\left(x_{n}\right)
\end{array}\right]^{T}
\end{aligned}
$$

where $\dot{V}_{i}=d V_{i} / d t$ for the trajectories $x_{i}(t) \in \mathbb{R}^{N_{i}}$. Then, the dissipation inequalities (1) can be compactly written as

$$
\dot{V}(x) \leq(-A+\Gamma) \circ V(x)+\Gamma_{r}(|r|) .
$$

Recall that the relation $\leq$ for vectors used in (2) is interpreted componentwise. The goal of this paper is to find a function $V_{c l}: \mathbb{R}^{N} \rightarrow \mathbb{R}_{+}$satisfying the dissipation inequality

$$
\dot{V}_{c l}(x) \leq-\alpha_{c l}(V(x))+\gamma_{c l}(|r|)
$$

along the trajectories $x(t)$ of the network $\Sigma$ for some $\alpha_{c l} \in \mathcal{P}$ and $\gamma_{c l} \in \mathcal{K} \cup\{0\}$. The property (3) guarantees that the network $\Sigma$ is iISS with respect to input $r$ and state $x$. Furthermore, the network $\Sigma$ is ISS if $\alpha_{c l} \in \mathcal{K}_{\infty}$.

\section{A TRICK}

Consider $\mathcal{C}^{1}$ functions $W_{i}: \mathbb{R}^{N_{i}} \rightarrow \mathbb{R}_{+}$given by

$$
W_{i}\left(x_{i}\right)=\int_{0}^{V_{i}\left(x_{i}\right)} \lambda_{i}(\tau) d \tau, \quad i=1,2, \ldots, n
$$

for continuous functions $\lambda_{i}: \mathbb{R}_{+} \rightarrow \mathbb{R}_{+}$. We assume that

$$
\begin{aligned}
& \lambda_{i}\left(s_{i}\right)>0, \quad \forall s_{i} \in(0, \infty), \quad i=1,2, \ldots, n \\
& \int_{1}^{\infty} \lambda_{i}\left(s_{i}\right) d s_{i}=\infty, \quad i=1,2, \ldots, n \\
& \left\{\alpha_{i i} \in \mathcal{K} \backslash \mathcal{K}_{\infty} \wedge \gamma_{r, i} \in \mathcal{K} \Rightarrow \limsup _{s_{i} \rightarrow \infty} \lambda_{i}\left(s_{i}\right)<\infty\right\} \\
& \quad, \quad i=1,2, \ldots, n
\end{aligned}
$$

hold. We consider the operator $F: \mathbb{R}_{+}^{n} \rightarrow \mathbb{R}_{+}^{n}$ defined as

$$
F(s)=\left[\zeta_{1}\left(s_{1}\right), \zeta_{2}\left(s_{2}\right), \ldots, \zeta_{n}\left(s_{n}\right)\right]^{T}
$$

where we assume that

$$
\zeta_{i} \in \mathcal{K}_{\infty}, \quad \mathbf{I d}-\zeta_{i} \in \mathcal{K}_{\infty}, \quad i=1,2, \ldots, n
$$

The functions $\lambda_{i}$ and $\zeta_{i}$ have yet to be determined.

Using these functions, we define the vectors

$$
\begin{aligned}
& W(x)=\left[\begin{array}{lll}
W_{1}\left(x_{1}\right), & W_{2}\left(x_{2}\right), \ldots, & W_{n}\left(x_{n}\right)
\end{array}\right]^{T} \\
& \dot{W}(x)=\left[\begin{array}{lll}
\dot{W}_{1}\left(x_{1}\right), & \dot{W}_{2}\left(x_{2}\right), \ldots, & \dot{W}_{n}\left(x_{n}\right)
\end{array}\right]^{T}
\end{aligned}
$$

along the trajectories $x_{i}(t)$ and the matrices

$$
\begin{aligned}
& H(V(x))=\left[\begin{array}{cccc}
\lambda_{1}\left(V_{1}\left(x_{1}\right)\right) & 0 & \cdots & 0 \\
0 & \lambda_{2}\left(V_{2}\left(x_{2}\right)\right) & \ddots & \vdots \\
\vdots & \ddots & \ddots & 0 \\
0 & \cdots & 0 & \lambda_{n}\left(V_{n}\left(x_{n}\right)\right)
\end{array}\right] \\
& G(|r|)=\left[\begin{array}{cccc}
\eta_{1}(|r|) & 0 & \cdots & 0 \\
0 & \eta_{2}(|r|) & \ddots & \vdots \\
\vdots & \ddots & \ddots & 0 \\
0 & \cdots & 0 & \eta_{n}(|r|)
\end{array}\right]
\end{aligned}
$$

where the non-decreasing continuous functions $\eta_{i}: \mathbb{R}_{+} \rightarrow$ $\mathbb{R}_{+}, i=1,2, \ldots, n$, are given by

$$
\begin{aligned}
& \bar{\lambda}_{i}(\tau)=\max _{w \in[0, \tau]} \lambda_{i}(w) \\
& \eta_{i}(\tau)=\left\{\begin{aligned}
\bar{\lambda}_{i} \circ \alpha_{i i}^{-1} \circ \zeta_{i}^{-1} \circ \gamma_{r, i}(\tau) \\
, \text { if } \lim _{w \rightarrow \infty} \zeta_{i} \circ \alpha_{i i}(w)>\gamma_{r, i}(\tau) \\
\lim _{w \rightarrow \infty} \bar{\lambda}_{i}(w), \text { otherwise }
\end{aligned}\right.
\end{aligned}
$$

Note that the assumption (7) renders the function $\eta_{i}: \mathbb{R}_{+} \rightarrow$ $\mathbb{R}_{+}$given by (10) well-defined. With the help of these definitions, combining the two cases $\zeta_{i} \propto \alpha_{i i}\left(V_{i}\left(x_{i}\right)\right)>\gamma_{r, i}(|r|)$ and $\zeta_{i} \circ \alpha_{i i}\left(V_{i}\left(x_{i}\right)\right) \leq \gamma_{r, i}(|r|)$ in (2) proves that (2) implies

$$
\begin{aligned}
\dot{W}(x) \leq H(V(x))\{-(\mathbf{I d}-F) \circ A & +\Gamma\}(V(x)) \\
& +G(|r|) \Gamma_{r}(|r|)(11)
\end{aligned}
$$


Alternatively, the inequality (11) can be expressed as

$$
\begin{array}{r}
\dot{W}(x) \leq H(V(x))\left\{-(\mathbf{I d}+E)^{-1} \circ A+\Gamma\right\}(V(x)) \\
+G(|r|) \Gamma_{r}(|r|)(12)
\end{array}
$$

where $E$ is defined by

$$
\begin{aligned}
(\mathbf{I d}+E)(s) & =\left[s_{1}+\varepsilon_{1}\left(s_{1}\right), s_{2}+\varepsilon_{2}\left(s_{2}\right), \ldots, s_{n}+\varepsilon_{n}\left(s_{n}\right)\right]^{T} \\
& =(\mathbf{I d}-F)^{-1}(s)
\end{aligned}
$$

Note that $\varepsilon_{i} \in \mathcal{K}_{\infty}$ holds since

$$
\begin{aligned}
\left(\mathbf{I d}+\varepsilon_{i}\right) & \circ\left(s_{i}-\zeta_{i}\left(s_{i}\right)\right)-s_{i} \\
& =-\zeta_{i}\left(s_{i}\right)+\varepsilon_{i} \circ\left(s_{i}-\zeta_{i}\left(s_{i}\right)\right)=0
\end{aligned}
$$

and $\zeta_{i}$, Id $-\zeta_{i} \in \mathcal{K}_{\infty}$. The relation (13) defines a bijection between $\zeta_{i} \in \mathcal{K}_{\infty}$ and $\varepsilon_{i} \in \mathcal{K}_{\infty}$, i.e., $F$ and $E$.

The technique applied to the iISS network in this section is essentially the same as the technique of changing ISS supply rates proposed in [20].

Remark 2: The choice of $\zeta_{i}(s) \equiv 0, \varepsilon_{i}(s) \equiv 0$ and $\eta_{i}(s) \equiv 0$ is also valid when $\gamma_{r, i}(s) \equiv 0$.

\section{SUM-Type Construction}

This sections presents a condition under which the network $\Sigma$ is guaranteed to be iISS. For this purpose, we seek Lyapunov functions in the form of

$$
V_{c l}(x)=\sum_{i=1}^{n} W_{i}\left(x_{i}\right)
$$

In order to select functions $\lambda_{i}$ with which the sum-type Lyapunov function establishes the stability of the network, we define mappings from $s \in \mathbb{R}_{+}^{n}$ to $\mathbb{R}_{+}^{n}$ by

$$
\begin{aligned}
& \Lambda(s)=\left[\lambda_{1}\left(s_{1}\right), \lambda_{2}\left(s_{2}\right), \ldots, \lambda_{n}\left(s_{n}\right)\right]^{T} \\
& D(s)=\left[s_{1}+\beta_{1}\left(s_{1}\right), s_{2}+\beta_{2}\left(s_{2}\right), \ldots, s_{n}+\beta_{n}\left(s_{n}\right)\right]^{T}
\end{aligned}
$$

and obtain the following theorem.

Theorem 1: Suppose that there exist continuous functions $\lambda_{i}: \mathbb{R}_{+} \rightarrow \mathbb{R}_{+}, i=1,2, \ldots, n$, such that (5), (6) (7) and

$$
\Lambda(s)^{T} \Gamma(s) \leq \Lambda(s)^{T} D^{-1} \circ A(s), \quad \forall s \in \mathbb{R}_{+}^{n}
$$

are satisfied for some $\beta_{1}, \beta_{2}, \ldots, \beta_{n} \in \mathcal{K}_{\infty}$. Then the network $\Sigma$ is iISS with respect to input $r$ and state $x$. If

$$
\begin{aligned}
& \alpha_{i i} \in \mathcal{K}_{\infty}, \quad i=1,2, \ldots, n \\
& \liminf _{s_{i} \rightarrow \infty} \lambda_{i}\left(s_{i}\right)>0, \quad i=1,2, \ldots, n
\end{aligned}
$$

are satisfied additionally, the network $\Sigma$ is ISS. Furthermore, an iISS (ISS) Lyapunov function is given by (14).

Proof: Substituting (17) for (12), we obtain

$\dot{V}_{c l}(x) \leq-\Lambda(V)^{T}\left[\left((\mathbf{I d}+E)^{-1}-D^{-1}\right) \circ A(V)\right]+\sum_{i=1}^{n} \hat{\gamma}_{r, i}(|r|)$

where $\hat{\gamma}_{r, i}:=\eta_{i} \gamma_{r, i} \in \mathcal{K} \cup\{0\}$. Let $\theta_{i}$ be defined with

$$
\mathbf{I d}-\theta_{i}=\left(\mathbf{I d}+\beta_{i}\right)^{-1}
$$

The property $\theta_{i} \in \mathcal{K}_{\infty}$ follows from $\beta_{i} \in \mathcal{K}_{\infty}$ and

$$
\begin{aligned}
\left(\mathbf{I d}-\theta_{i}\right) & \circ\left(\mathbf{I d}+\beta_{i}\right)\left(s_{i}\right)-s_{i} \\
& =\beta_{i}\left(s_{i}\right)-\theta_{i} \circ\left(\mathbf{I d}+\beta_{i}\left(s_{i}\right)\right)=0
\end{aligned}
$$

Pick $\zeta_{i} \in \mathcal{K}_{\infty}$ satisfying $\theta_{i}-\zeta_{i} \in \mathcal{K}_{\infty}$. Then, from

$$
\left(\mathbf{I d}+\varepsilon_{i}\right)^{-1}-\left(\mathbf{I d}+\beta_{i}\right)^{-1}=\theta_{i}-\zeta_{i}
$$

we obtain

$$
\begin{array}{r}
\dot{V}_{c l}(x) \leq-\sum_{i=1}^{n} \lambda_{i}\left(V_{i}\left(x_{i}\right)\right)\left[\left(\theta_{i}-\zeta_{i}\right) \circ \alpha_{i i}\left(V_{i}\left(x_{i}\right)\right)\right] \\
+\sum_{i=1}^{n} \hat{\gamma}_{r, i}(|r|)
\end{array}
$$

Since $\alpha_{i i} \in \mathcal{K}$ implies $\left(\theta_{i}-\zeta_{i}\right) \circ \alpha_{i i} \in \mathcal{K}$, we have

$$
\dot{V}_{c l}(x) \leq-\sum_{i=1}^{n} \hat{\alpha}_{i}\left(V_{i}\left(x_{i}\right)\right)+\sum_{i=1}^{n} \hat{\gamma}_{r, i}(|r|)
$$

for some $\hat{\alpha}_{i} \in \mathcal{P}, i=1,2, . ., n$, so that we can arrive at (3). The properties (5) and (6) ensure that the functions $W_{i}$ are positive definite and radially unbounded. Thus, $V_{c l}$ is an iISS Lyapunov function of the network $\Sigma$. If (18) and (19) hold additionally, we obtain $\left(\theta_{i}-\zeta_{i}\right) \circ \alpha_{i i} \in \mathcal{K}_{\infty}$ and $\hat{\alpha}_{i} \in \mathcal{K}_{\infty}$, which implies that $V_{c l}$ is an ISS Lyapunov function.

In the case of $n=2$, we can obtain a solution $\Lambda(s)$ fulfilling all the requirements in Theorem 1 as explained in the following.

Theorem 2: Let $n=2$. Suppose that

$$
\left\{\alpha_{i i} \in \mathcal{K} \backslash \mathcal{K}_{\infty} \Rightarrow \gamma_{3-i, i} \in \mathcal{K} \backslash \mathcal{K}_{\infty} \cup\{0\}\right\}, i=1,2
$$

holds. If there exist $\beta_{1}, \beta_{2} \in \mathcal{K}_{\infty}$ satisfying

$$
D \circ \Gamma(s) \nsupseteq A(s), \quad \forall s \in \mathbb{R}_{+}^{2} \backslash\{0\}
$$

there exists a solution $\Lambda(s)$ fulfilling all the requirements in Theorem 1.

Proof: It can be verified that the condition (23) is equivalent to the logical sum of

$$
\begin{array}{r}
\left(\mathbf{I d}+\beta_{1}\right) \circ \gamma_{12} \circ \alpha_{22}^{-1} \circ\left(\mathbf{I d}+\beta_{2}\right) \circ \gamma_{21}(\tau)<\alpha_{11}(\tau), \\
\forall \tau \in(0, \infty)(24)
\end{array}
$$

and

$$
\begin{array}{r}
\left(\mathbf{I d}+\beta_{2}\right) \circ \gamma_{21} \circ \alpha_{11}^{-1} \circ\left(\mathbf{I d}+\beta_{1}\right) \circ \gamma_{12}(\tau)<\alpha_{22}(\tau), \\
\forall \tau \in(0, \infty)(25)
\end{array}
$$

Note that (24) ((25)) implicitly requires $\lim _{\tau \rightarrow \infty} \alpha_{22}(\tau) \geq$ $\lim _{\tau \rightarrow \infty} \gamma_{21}(\tau)\left(\lim _{\tau \rightarrow \infty} \alpha_{11}(\tau) \geq \lim _{\tau \rightarrow \infty} \gamma_{12}(\tau)\right.$, respectively). The existence of $\beta_{1}, \beta_{2} \in \mathcal{K}_{\infty}$ achieving the above logical sum is the same as the existence of $\bar{\beta}_{1}, \bar{\beta}_{2} \in \mathcal{K}_{\infty}$ achieving $\leq$ for $s \in \mathbb{R}_{+}$instead of $<$for $s \in \mathbb{R}_{+} \backslash\{0\}$ in the logical sum of (24) and (25). Indeed, the substitution $\beta_{i}=$ $\bar{\beta}_{i} / 2$ allows us to change $\leq$ into $<$. Hence, the condition (23) is equivalent to the iISS small-gain condition presented in [14]. The function $V_{c l}$ in (14) is identical with the one employed in [11], [14], and the corresponding inequality (17) is the same as the one solved in [11], [14]. Moreover, the property (22) implies that one of the properties
(A1) $\lim _{\tau \rightarrow \infty} \alpha_{11}(\tau)=\infty \wedge \lim _{\tau \rightarrow \infty} \alpha_{22}(\tau)=\infty$,
(A2) $\lim _{\tau \rightarrow \infty} \alpha_{11}(\tau)=\infty \wedge \lim _{\tau \rightarrow \infty} \gamma_{12}(\tau)<\infty$,
(A3) $\lim _{\tau \rightarrow \infty} \alpha_{22}(\tau)=\infty \wedge \lim _{\tau \rightarrow \infty} \gamma_{21}(\tau)<\infty$,
(A4) $\lim _{\tau \rightarrow \infty} \gamma_{12}(\tau)<\infty \wedge \lim _{\tau \rightarrow \infty} \gamma_{21}(\tau)<\infty$,

is satisfied. It is also verified that

$$
\begin{aligned}
& (24) \wedge(A 2) \wedge \neg(A 1) \Rightarrow(A 4) \\
& (25) \wedge(A 3) \wedge \neg(A 1) \Rightarrow(A 4)
\end{aligned}
$$


hold. Hence, the non-decreasing functions $\lambda_{1}\left(s_{1}\right)$ and $\lambda_{2}\left(s_{2}\right)$ derived in [14] achieve all the requirements in Theorem 1 for $n=2$. If $\gamma_{i, j}\left(s_{j}\right) \equiv 0$ holds for some $i \neq j$, we can always use sufficiently small $\gamma_{i, j} \in \mathcal{K}$ when we invoke [14].

In the $n=2$ case, the components $\lambda_{1}\left(s_{1}\right)$ and $\lambda_{2}\left(s_{2}\right)$ of $\Lambda(s)$ are derived explicitly in [11], [14]. For $n \geq 3$, no formula for solutions $\Lambda(s)$ to the problem posed in Theorem 1 has yet been known.

Remark 3: When we only consider 0-GAS (i.e., global asymptotic stability of $x=0$ for $r(t) \equiv 0$ or $\Gamma_{r}(s) \equiv 0$ ), The functions $\beta_{i}$ in Theorem 1 are only required to satisfy $\beta_{i} \in \mathcal{P}$ and $\mathbf{I d}+\beta_{i} \in \mathcal{K}_{\infty}$ for $i=1,2, \ldots, n$. Note that using $<$ in (17) with $D=$ Id cannot always ensure 0-GAS since it cannot exclude the no-gap case, [1]. It is known that in the no-gap case information on $\alpha_{i i}$ and $\gamma_{i j}$ is not sufficient to conclude 0-GAS. Property (17) with positive definite $\beta_{i}$ 's ensures that the no-gap case does not occur. Likewise, inequality (23) with $D=\mathbf{I d}$ cannot guarantee the 0 -GAS. In order to avoid the no-gap case, we need to add an assumption as in [14].

\section{MAX-TYPE CONSTRUCTION}

Define a locally Lipschitz function $V_{c l}: \mathbb{R}^{N} \rightarrow \mathbb{R}_{+}$by

$$
V_{c l}(x)=\max _{i=1,2, \ldots, n} W_{i}\left(x_{i}\right)
$$

Alternatively, we can write the above $V_{c l}$ as

$$
V_{c l}(x)=\max _{i=1,2, \ldots, n} \psi_{i}^{-1}\left(V_{i}\left(x_{i}\right)\right)
$$

where $\psi_{i} \in \mathcal{K}_{\infty}$ is given by

$$
\psi_{i}^{-1}\left(s_{i}\right)=\int_{0}^{s_{i}} \lambda_{i}(\tau) d \tau
$$

Note that the right hand side of the above equation is guaranteed to be of class $\mathcal{K}_{\infty}$ by (5) and (6). An apparent feature of the max-tye Lyapunov function (26) is its Lipschitz continuity, while the sum-type Lyapunov function (14) is continuously differentiable.

For interconnected ISS systems, some studies derive Lyapunov functions of the form (27), e.g., [15], [6], [18]. The following theorem demonstrates that the max-type Lyapunov function is not useful if at least one subsystem is only iISS.

Theorem 3: Let $V_{c l}$ be defined by (27), and let $V_{c l}^{\circ}(x ; \dot{x})$ denote the Clarke generalized derivative at $x$ in the direction of $\dot{x}$. If there exist continuously differentiable $\psi_{i} \in \mathcal{K}_{\infty}, i=$ $1,2 \ldots, n$, such that all differentiable trajectories ${ }^{1} x(t) \in \mathbb{R}^{N}$ fulfilling (1) with $\alpha_{i i} \in \mathcal{K}, \gamma_{i j} \in \mathcal{K} \cup\{0\}$ for $r(t) \equiv 0$ satisfy

$$
V_{c l}^{\circ}(x ; \dot{x}) \leq 0, \quad \forall x \in \mathbb{R}^{N},
$$

then

$$
\sum_{j \neq i} \lim _{\tau \rightarrow \infty} \gamma_{i j}(\tau) \leq \lim _{\tau \rightarrow \infty} \alpha_{i i}(\tau), \quad i=1,2, \ldots, n
$$

Proof: To prove the claim by contradiction, suppose that

$$
\sum_{j \neq i} \lim _{\tau \rightarrow \infty} \gamma_{i j}(\tau)>\lim _{\tau \rightarrow \infty} \alpha_{i i}(\tau)
$$

\footnotetext{
${ }^{1}$ Here, the trajectories are not necessarily associated with differential equations of the form $\dot{x}=f(x, r)$. Using the technique developed in [13], we can also address the existence of a corresponding differential equation in Theorem 3.
}

holds for some $i=p \in\{1,2, \ldots, n\}$. Let

$$
\begin{aligned}
& M_{p}:=\left\{x \in \mathbb{R}^{N}: \psi_{p}^{-1}\left(V_{p}\left(x_{p}\right)\right)>\psi_{j}^{-1}\left(V_{j}\left(x_{j}\right)\right), \forall j \neq p\right\} \\
& L_{p}:=\left\{x \in \mathbb{R}^{N}: \sum_{j \neq p} \gamma_{p j}\left(V_{j}\left(x_{j}\right)\right)>\lim _{\tau \rightarrow \infty} \alpha_{p p}(\tau)\right\}
\end{aligned}
$$

Since the $\psi_{i}^{-1}$,s are of class $\mathcal{K}_{\infty}$, the set $M_{p}$ is unbounded in all directions, i.e., $M_{p}$ contains a sequence $\left\{x^{p, k} \in \mathbb{R}^{N}\right\}$, $k=1,2, \ldots$, such that $V_{i}\left(x_{i}^{p, k}\right) \rightarrow \infty$ for all $i=1,2, \ldots, n$ when $k \rightarrow \infty$. This fact and (31) ensure $M_{p} \cap L_{p} \neq \emptyset$. Property (1) with $r(t) \equiv 0$ yields $\dot{V}_{c l}(x) \leq \xi(x)$ for $x \in M_{p}$, where

$$
\xi(x):=\lambda_{p}\left(V_{p}\left(x_{p}\right)\right)\left\{-\alpha_{p p}\left(V_{p}\left(x_{p}\right)\right)+\sum_{j \neq p} \gamma_{p j}\left(V_{j}\left(x_{j}\right)\right)\right\}
$$

By assumption, in the set $M_{p}$, the function $\xi(x)$ is the smallest upper bound of $\dot{V}_{c l}(x)$ covering all trajectories $x(t) \in \mathbb{R}^{N}$ defined with (1). The definition of $L_{p}$ implies

$$
\xi(x)>0, \quad \forall x \in M_{p} \cap L_{p}
$$

Although $V_{c l}$ defined in (27) is only locally Lipschitz, it is differentiable on $M_{p} \cap L_{p}$. Since the Clarke generalized derivative agrees with the directional derivative of $V_{c l}$ at differentiable points. the property (32) contradicts (29).

The property (30) means that each subsystem $\Sigma_{i}$ is ISS with respect to input $x_{j}, j \neq i$ and state $x_{i}$ [21]. Theorem 3 can be interpreted as follows: In the construction of a Lyapunov function of the form (27), the function $\psi_{i}^{-1}$ needs to ensure that if the maximum of (27) is attained for the $i$-th subsystem, then the decay of the particular subsystem appears as the decrease of the function $V_{c l}$. Thus, the max-type construction requires that each subsystem be contractive when its state is large. However, this property is not guaranteed when a subsystem is iISS.

If we restrict our attention to networks of ISS subsystems. we can derive stability based on the max-type Lyapunov function. Using the mapping from $\mathbb{R}_{+} \rightarrow \mathbb{R}_{+}^{n}$ defined by

$$
\Psi(\tau)=\left[\psi_{1}(\tau), \psi_{2}(\tau), \ldots, \psi_{n}(\tau)\right]^{T}
$$

the following demonstrates this fact.

Theorem 4: Suppose that there exist continuous functions $\lambda_{i}: \mathbb{R}_{+} \rightarrow \mathbb{R}_{+}, i=1,2, \ldots, n$, such that (5), (6) (7) and

$$
D \circ \Gamma(\Psi(\tau)) \leq A(\Psi(\tau)), \quad \forall \tau \in \mathbb{R}_{+}
$$

are satisfied for some $\beta_{1}, \beta_{2}, \ldots, \beta_{n} \in \mathcal{K}_{\infty}$. Then, the network $\Sigma$ is iISS with respect to input $r$ and state $x$. If (18) and (19) are satisfied additionally, the network $\Sigma$ is ISS. Furthermore, an iISS (ISS) Lyapunov function is given by (26).

Proof: Suppose that $\psi_{i} \in \mathcal{K}_{\infty}, i=1,2, \ldots, n$ fulfill all the requirements in Theorem 4. Assume for the moment that, for $x \neq 0$, the maximum in (26) is attained uniquely by the $i=p \in\{1,2, \ldots, n\}$, i.e.,

$$
\psi_{p}^{-1}\left(V_{p}\left(x_{p}\right)\right)>\psi_{j}^{-1}\left(V_{j}\left(x_{j}\right)\right), \quad \forall j \neq p
$$

Let $[\Gamma(s)]_{p}$ denote the $p$-th component of the vector $\Gamma(s)$. Then, for $V_{c l}(x)$ defined in (26), the inequality (12) yields

$$
\begin{array}{r}
\dot{V}_{c l}(x) \leq \lambda_{p}\left(V_{p}\left(x_{p}\right)\right)\left\{-\left(\mathbf{I d}+\varepsilon_{p}\right)^{-1} \alpha_{p p}\left(V_{p}\right)\right. \\
\left.+[\Gamma(V)]_{p}\right\}+\eta_{p}(|r|) \gamma_{r, p}(|r|)
\end{array}
$$


Since the definition of $\Gamma$ and (35) ensure

$$
\begin{aligned}
{[\Gamma(V)]_{p} } & =\left[\Gamma\left(\left[\psi_{1} \circ \psi_{1}^{-1}\left(V_{1}\left(x_{1}\right)\right), \ldots, \psi_{n} \circ \psi_{n}^{-1}\left(V_{n}\left(x_{n}\right)\right)\right]\right)\right]_{p} \\
& \leq\left[\Gamma\left(\Psi \circ \psi_{p}^{-1}\left(V_{p}\left(x_{p}\right)\right)\right)\right]_{p},
\end{aligned}
$$

we obtain

$$
\begin{aligned}
\dot{V}_{c l}(x) & \leq \lambda_{p}\left(V_{p}\left(x_{p}\right)\right)\left\{-\left(\mathbf{I d}+\varepsilon_{p}\right)^{-1} \circ \alpha_{p p}\left(\psi_{p} \circ \psi_{p}^{-1}\left(V_{p}\left(x_{p}\right)\right)\right)\right. \\
+ & {\left.\left[\Gamma\left(\Psi \circ \psi_{p}^{-1}\left(V_{p}\left(x_{p}\right)\right)\right)\right]_{p}\right\}+\eta_{p}(|r|) \gamma_{r, p}(|r|) }
\end{aligned}
$$

from the definition of $A$. Now, let $\theta_{p} \in \mathcal{K}_{\infty}$ be computed with (20). Pick $\zeta_{p} \in \mathcal{K}_{\infty}$ satisfying $\theta_{p}-\zeta_{p} \in \mathcal{K}_{\infty}$. From the $p$-th row of (34), $\psi_{p} \in \mathcal{K}_{\infty}$ and (21) it follows that

$$
\dot{V}_{c l}(x) \leq-\lambda_{p}\left(V_{p}\left(x_{p}\right)\right)\left[\left(\theta_{p}-\zeta_{p}\right) \circ \alpha_{p p}\left(V_{p}\left(x_{p}\right)\right)\right]+\hat{\gamma}_{r, p}(|r|)
$$

holds for $\hat{\gamma}_{r, p}:=\eta_{p} \gamma_{r, p} \in \mathcal{K} \cup\{0\}$. Therefore, there exists $\hat{\alpha}_{i} \in \mathcal{P}$ such that

$$
\dot{V}_{c l}(x) \leq-\hat{\alpha}_{p}\left(V_{p}\left(x_{p}\right)\right)+\hat{\gamma}_{r, p}(|r|)
$$

is satisfied. The functions $\left(\theta_{p}-\zeta_{p}\right) \circ \alpha_{p p}$ and $\hat{\alpha}_{p}$ are of class $\mathcal{K}_{\infty}$ if (18) and (19) hold. Repeating (37) for $p \in\{1,2, \ldots, n\}$ and using $V_{c l}(x)=\psi_{p}^{-1}\left(V_{p}\left(x_{p}\right)\right)$ implied by (35), we have

$$
\dot{V}_{c l}(x) \leq-\max _{i} \hat{\alpha}_{i} \circ \psi_{i}\left(V_{c l}(x)\right)+\max _{i} \hat{\gamma}_{r, i}(|r|)
$$

for all $x \in \mathbb{R}^{N}$ where the maximization in (26) is uniquely defined. The set of such points is an open and dense in $\mathbb{R}^{N}$. For the rest of the proof, we can employ the arguments in [4], [3], [8]. Since the locally Lipschitz continuous function $V_{c l}$ is the maximization of $\mathcal{C}^{1}$ functions $V_{i}$, the Clarke subgradient of $V_{c l}$ in $x \in \mathbb{R}^{n}$ can be computed by the set

$$
\partial_{C l} V_{c l}(x)=\operatorname{conv}\left\{\begin{array}{c}
\nabla \\
\left.\left.\sigma_{i}^{-1} \circ \alpha_{i}^{-1} \circ V_{i}\left(x_{i}\right)\right)=V_{c l}(x)\right\},
\end{array}\right.
$$

where conv $\{\cdot\}$ denotes the convex hull. As we have (38) for each of the extremal points of $\partial_{C l} V_{c l}(x)$, the dissipation inequality (38) holds in terms of the Clarke generalized derivative for each $\zeta$ in the Clarke subgradient. Thus, the function $V_{c l}$ given in (26) is a Lipschitz continuous iISS (ISS) Lyapunov function for the network $\Sigma$.

It is stressed that since $\psi_{i}$ 's are class $\mathcal{K}_{\infty}$ functions, the condition (34) implies (30). This fact is consistent with Theorem 3. Theorem 4 does not require the $\alpha_{i i}$ 's to be of class $\mathcal{K}_{\infty}$ which are assumed in [15], [8], [18]. Although both Theorem 4 and the results in [15], [8], [18] deal with ISS subsystems, Theorem 4 allows us to get rid of transformation into $\alpha_{i i} \in \mathcal{K}_{\infty}$ which gives rise to unnecessary conservativeness in practice. In contrast to Theorem 4 of the max type, the sum-type construction presented in Section IV can deal with iISS subsystems which are not ISS. The limiting value of (17) does not result in a restriction like (30) since the parameter $\Lambda(s)$ is "multiplied" on both sides of (17). In fact, in the case of $n=2$, the inequality (24) can be satisfied even if $\gamma_{12}(\infty)>\alpha_{11}(\infty)$ as long as $\gamma_{21}(\infty)<$ $\alpha_{22}(\infty)$. In the same way, the inequality (25) can be satisfied even if $\gamma_{21}(\infty)>\alpha_{22}(\infty)$ as long as $\gamma_{12}(\infty)<\alpha_{11}(\infty)$. Note that, to obtain iISS of $\Sigma$, some of the subsystems $\Sigma_{i}$ is necessarily ISS but not all, which is proved in [13].

Now, we address the existence of solutions $\Psi$ to (34). The following theorem presents a condition guaranteeing the existence, which is a consequence of the results developed in [8], [18].

Theorem 5: Assume that $\alpha_{i i}, i=1,2, \ldots, n$, are of class $\mathcal{K}_{\infty}$ and $\mathcal{C}^{1}$, and that $\Gamma$ is irreducible, [8]. Suppose that there exist $\beta_{1}, \beta_{2}, \ldots, \beta_{n} \in \mathcal{K}_{\infty}$ satisfying

$$
D \circ \Gamma(s) \nsupseteq A(s), \quad \forall s \in \mathbb{R}_{+}^{n} \backslash\{0\}
$$

Then, there exist continuously differentiable functions $\psi_{i} \in$ $\mathcal{K}_{\infty}, i=1,2, \ldots, n$ such that (34) and

$$
\begin{aligned}
& \frac{d}{d \tau} \psi_{i}(\tau)>0, \quad \forall \tau \in(0, \infty), \quad i=1,2, \ldots, n \\
& \limsup _{\tau \rightarrow \infty} \frac{d}{d \tau} \psi_{i}(\tau)<\infty, \quad i=1,2, \ldots, n
\end{aligned}
$$

are satisfied.

Proof: By virtue of $\alpha_{i i} \in \mathcal{K}$, (39) is equivalent to

$$
D \circ \Gamma \circ A^{-1}(s) \nsupseteq s, \quad \forall s \in \mathbb{R}_{+}^{n} \backslash\{0\}
$$

The results in [8], [18], [9] with smoothing guarantees the existence of $\mathcal{C}^{1}$ functions $\hat{\psi}_{i} \in \mathcal{K}_{\infty}, i=1,2, \ldots, n$ satisfying

$$
\begin{aligned}
& D \circ \Gamma \circ A^{-1}(\hat{\Psi}(\tau))<\hat{\Psi}(\tau), \quad \forall \tau \in(0, \infty) \\
& \frac{d}{d \tau} \hat{\psi}_{i}(\tau)>0, \quad \forall \tau \in(0, \infty), \quad i=1,2, \ldots, n \\
& \limsup _{\tau \rightarrow \infty} \frac{d}{d \tau} \hat{\psi}_{i}(\tau)<\infty, \quad i=1,2, \ldots, n
\end{aligned}
$$

where $\hat{\Psi}=\left[\hat{\psi}_{1}, \hat{\psi}_{2}, \ldots, \hat{\psi}_{n}\right]^{T}$. Note that if (45) is not satisfied by a particular $\hat{\Psi}$, we can always find a continuously differentiable $\rho \in \mathcal{K}_{\infty}$ such that replacing $\hat{\Psi}$ by $\hat{\Psi}(\rho)$ achieves (43), (44), and (45). Setting $\Psi(\tau)=A^{-1} \circ \hat{\Psi}(\tau)$, we arrive at (34). The property (44) and the differentiability of $\alpha_{i i}$ ensure (40). The property (41) follows from (45).

Note that the properties $\psi_{i} \in \mathcal{K}_{\infty}, i=1,2, \ldots, n$, and (40) imply (5) and (6). The property (41) ensures (19). Hence, the above theorem guarantees the existence of solutions $\left\{\lambda_{i}\right\}$ to the problem posed by Theorem 4 in the case of $\alpha_{11}, \ldots, \alpha_{n n} \in \mathcal{K}_{\infty}$. Jiang et al.[15] proved Theorem 5 in the case where the network $\Sigma$ consists of two ISS subsystems.

The condition (39) is identical to (23) for $n=2$. According to Theorem 3, the gap between (39) and (34) requiring $\psi_{i} \in \mathcal{K}_{\infty}$ is larger than the one between (39) and (17) when the subsystems are only iISS.

In the case of linear $A, \Gamma, D$, both the problems posed in Theorem 1 and Theorem 4 can be solved by theorems of the Perron-Frobenius type. A necessary and sufficient condition for the solvability is $\rho\left(\Gamma A^{-1}\right)<1$, where $\rho(\cdot)$ denotes the spectral radius[5]. The functions $\Lambda$ and $\Psi$ are obtained as a suitable lefteigenvector and a right eigenvector, respectively.

Remark 4: When we only consider 0-GAS, the condition (34) can be replaced by $\Gamma(\Psi(\tau))<A(\Psi(\tau)), \forall \tau \in \mathbb{R}_{+} \backslash\{0\}$.

\section{Two ISS Subsystems CASE}

In (14) and (26) two different ways for the construction of Lyapunov functions for $\Sigma$ are presented. Because of the difference in the construction we cannot expect to have simple relations between conditions under which the composed functions $V_{c l}$ computed by (14) and (26) serve as iISS/ISS Lyapunov functions of the network. Nevertheless, if 
we restrict our attention to networks of two ISS subsystems, some relations between the sum and the max formulations can be obtained. The following is a direct consequence of Theorems 2 and 5.

Proposition 1: Let $n=2$. Assume $\alpha_{i i} \in \mathcal{K}_{\infty} \cap \mathcal{C}^{1}$ for $i=1,2$. Suppose that there exist $\beta_{1}, \beta_{2} \in \mathcal{K}_{\infty}$ satisfying (23). Then, the following hold:

(i) There exist continuous functions $\lambda_{1}, \lambda_{2}: \mathbb{R}_{+} \rightarrow \mathbb{R}_{+}$ such that (5), (6) (17) and (19) are satisfied.

(ii) There exist continuous functions $\psi_{1}, \psi_{2} \in \mathcal{K}_{\infty}$ such that (34) and (19) are satisfied.

Thus, under the unified condition (23), we can obtain ISS Lyapunov functions for the network $\Sigma$ based on the two approaches. The extension of this fact to the general $n$ subsystems case has not yet been accomplished. Proposition 1 has been first demonstrated for the linear case in [5].

In the rest of this section, we relate the solution $\Lambda$ in the max-type construction to the solution $\Psi$ in the sum-type construction in the $n=2$ case. For simplicity, we consider

$$
\beta_{i}(\tau)=c_{i} \tau, \quad i=1,2
$$

The following theorem constructs an $\mathcal{C}^{1}$ iISS Lyapunov function for $\Sigma$ by exploiting the solution in the max-type construction, which results in $\lambda_{i}$ 's different from [11], [14].

Theorem 6: Let $n=2$. Assume $\alpha_{11}, \alpha_{22} \in \mathcal{K}_{\infty}$. Suppose that there exist $\psi_{1}, \psi_{2} \in \mathcal{K}_{\infty}$ such that (34) is satisfied with (46) for some $c_{1}, c_{2}>2$. Then, the choice

$$
\begin{aligned}
& \Lambda(s)=\left[\begin{array}{c}
\lambda_{1}\left(s_{1}\right) \\
\lambda_{2}\left(s_{2}\right)
\end{array}\right]=\left[\begin{array}{c}
-P_{2} \circ \psi \circ \psi_{1}^{-1}\left(s_{1}\right) \\
-P_{1} \circ \psi \circ \psi_{2}^{-1}\left(s_{2}\right)
\end{array}\right] \\
& P(s)=\left[\begin{array}{c}
P_{1}(s) \\
P_{2}(s)
\end{array}\right]=-D_{H}^{-1} \circ A(s)+\Gamma(s), D_{H}(s)=\left[\begin{array}{c}
\frac{c_{1}}{2} s_{1} \\
\frac{c_{2}}{2} s_{2}
\end{array}\right]
\end{aligned}
$$

satisfies (5), (6) (17) and (19) with (46) for another pair of $c_{1}, c_{2}>1$.

Theorem 6 provides a new way to construct $\left\{\lambda_{1}, \lambda_{2}\right\}$ for the iISS Lyapunov function in the sum from (14) for $n=2$. Although different pairs of solutions are available in [11], [14], making a choice from many Lyapunov functions is sometimes advantageous in systems analysis and design. The solutions $\left\{\lambda_{1}, \lambda_{2}\right\}$ presented in [11], [14] are better than the solutions obtained through Theorem 6 in the sense not only that $c_{1}, c_{2}>2$ is not needed in [11], [14], but also that the solutions in [11], [14] can establish the stability of the network even when some subsystems are only iISS.

\section{CONCLUDING REMARKS}

This paper has demonstrated that the sum-type construction not only provides us with continuously differentiable Lyapunov functions directly, but also covers the class of iISS subsystems which are not ISS, while the max-type construction based on Lipschitz continuous Lyapunov functions requires the subsystems to be ISS. Solutions fulfilling the stability condition derived in the max-type construction are available in [15] for two subsystems, and [8], [18] for $n$ subsystems. In contrast, solving the stability condition in the sum-type construction has been harder, and formulas of solutions are only available in the case of $n=2$ [11], [14]. This situation seems natural since the sum-type construction deals with the broader class of systems. However, the relationship between the solutions in the sum-type construction and the max-type construction had not yet been known even in the case of ISS systems. This paper has shown several relations by unifying the solvability conditions and deriving a new solution to the sum-type construction from the solution to the max-type one the $n=2$ case.

\section{REFERENCES}

[1] D. Angeli and A. Astolfi, "A tight small gain theorem for not necessarily ISS systems," Syst. Control Lett., Vol. 56, pp. 87-91, 2007.

[2] D. Angeli, E.D. Sontag and Y. Wang, "A characterization of integral input-to-state stability," IEEE Trans. Autom. Control, Vol. 45, pp. 10821097, 2000.

[3] F.H. Clarke, "Nonsmooth analysis in control theory: a survey," European J. Contr., Vol. 7, pp.63-78, 2001.

[4] F.H. Clarke, Yu.S. Ledyaev, R.J. Stern, and P.R. Wolenski, Nonsmooth analysis and control theory, Springer-Verlag, Berlin, 1998.

[5] S. Dashkovskiy, H. Ito and F. Wirth, "On a small-gain theorem for ISS networks in dissipative Lyapunov form," Proc. 10th European Contr. Conf., pp.1077-1082, 2009.

[6] S. Dashkovskiy, B. Rüffer, and F. Wirth, "An ISS Lyapunov function for networks of ISS systems," Proc. 17th Int. Symp. Math. Theory of Networks and Systems, pp.77-82, 2006.

[7] S. Dashkovskiy, B. Rüffer, and F. Wirth, "An ISS small-gain theorem for general networks," Mathe. Contr. Signals and Syst., Vol. 19, pp.93122,2007

[8] S. Dashkovskiy, B.S. Rüffer, and F.R. Wirth, "A Lyapunov ISS small gain theorem for strongly connected networks," Proc. 7th IFAC Symp. Nonlinear Contr. Syst., pp.283-288, 2007.

[9] S. Dashkovskiy, B.S. Rüffer, and F.R. Wirth, "Small gain theorems for large scale systems and construction of ISS Lyapunov functions," submitted, 2009, Available online: http://arxiv.org/pdf/0901.1842.

[10] H. Ito, "A tool for stability analysis of nonlinear large-scale systems by cascading star products," American Control Conf., pp.632-637, 2006.

[11] H. Ito, "State-dependent scaling problems and stability of interconnected iISS and ISS systems," IEEE Trans. Automat. Contr., Vol. 51, pp.1626-1643, 2006.

[12] H. Ito, "A Lyapunov approach to integral input-to-state stability of cascaded systems with external signals," Proc. 47th IEEE Conf. Decision Contr., pp. 628-633, 2008.

[13] H. Ito and Z-P. Jiang, "Nonlinear small-gain condition covering iISS systems: Necessity and sufficiency from a Lyapunov perspective," Proc. 45th IEEE Conf. Decision Contr., pp. 355-360, 2006.

[14] H. Ito, and Z.-P. Jiang, "Small-gain conditions and Lyapunov functions applicable equally to iISS and ISS systems without uniformity assumption," Proc. 2008 American Contr. Conf., pp. 2297-2303, 2008.

[15] Z.P. Jiang, I. Mareels, and Y. Wang, "A Lyapunov formulation of the nonlinear small-gain theorem for interconnected ISS systems," Automatica, Vol.32, pp.1211-1215, 1996.

[16] Z-P. Jiang, A.R. Teel and L. Praly, "Small-gain theorem for ISS systems and applications," Mathe. Contr. Signals and Syst., Vol. 7, pp.95-120, 1994.

[17] Z-P. Jiang and Y. Wang, "A generalization of the nonlinear small-gain theorem for large-scale complex systems," Proc. 2008 World Congress on Intelligent Control and Automation, pp. 1188-1193, 2008.

[18] B.S. Rüffer, Monotone Systems, Graphs, and Stability of Large-Scale Interconnected Systems, Dissertation, Fachbereich 3, Mathematik und Informatik, University of Bremen, Germany, August 2007. Available online: http://nbn-resolving.de/urn:nbn:de:gbv:46-diss000109058.

[19] E.D. Sontag. "Smooth stabilization implies coprime factorization," IEEE Trans. Automat. Contr., Vol. 34, pp.435-443, 1989.

[20] E.D. Sontag and A.R. Teel, Changing supply functions in input/state stable systems, IEEE Trans. Automat. Contr., 40, pp. 1476-1478, 1995

[21] E.D. Sontag, and Y. Wang. "On characterizations of input-to-state stability property," Systems and Contr. Letters, 24, pp.351-359, 1995.

[22] A.R. Teel. "A nonlinear small gain theorem for the analysis of control systems with saturation," IEEE Trans. Automat. Contr., Vol. 41, pp.1256-1270, 1996. 